\title{
Administering antihypertensive drugs after acute ischemic stroke: timing is everything
}

$\mathrm{H}$ ypertension is a common early finding in patients who have experienced an acute ischemic stroke. It occurs both in patients who were normotensive and in those who were receiving antihypertensive therapy before the stroke. In many cases the hypertension that follows an ischemic stroke is transient, often lasting 24-48 hours. The blood pressure rise is due to 1 or more of the following mechanisms: impaired neurogenic cardiovascular control, autonomic dysregulation, baroreflex failure, increased sympathetic drive, reflex response to cerebral ischemia and mental stress.

Observational studies have shown a U-shaped relation between baseline blood pressure (immediately after the onset of the stroke) and likelihood of adverse clinical outcome. In one study the best prognosis was associated with a baseline systolic blood pressure of about $150 \mathrm{~mm} \mathrm{Hg},{ }^{1}$ whereas in another study the best prognosis was associated with a baseline systolic blood pressure of about $180 \mathrm{~mm} \mathrm{Hg}$ and the worst prognosis with a rapid fall of pressure. ${ }^{2}$ Despite these observations, the debate continues over whether such increases in blood pressure should be corrected early after stroke. The issue is complicated by the clinical heterogeneity of acute ischemic stroke, the complexity of the post-stroke physiological response and the rapidity of change of cerebral blood flow autoregulation after stroke onset.

Patients with lacunar infarcts - subcortical lesions more common in hypertensive $\approx$ and diabetic patients - tend to have milder neurologic deficits and higher baseline blood pressures but a better clinical outcome than patients with either atherothrombotic or cardioembolic stroke of the anterior or posterior circulation. ${ }^{3}$ Patients with lacunar infarcts more often die of cardiac complications, whereas those with atherothrombotic or cardioembolic stroke die of complications more directly related to the neurologic damage and immobilization. The better outcomes observed among patients with lacunar infarcts can likely be attributed to differences in lesion size, since such infarcts tend to result in smaller lesions and less damage than atherothrombotic and cardioembolic strokes. However, these outcomes also suggest that a high baseline blood pressure is not necessarily deleterious and may even be protective in some stroke patients.

When deciding whether to give antihypertensive drugs to reduce the blood pressure after stroke, we should distinguish the early phase (the first 24-48 hours) from the late phase because of the rapid changes of cerebral blood flow autoregulation that occur after stroke. In the healthy brain, cerebral blood flow is kept at $50 \mathrm{~mL} / 100 \mathrm{~g}$ per minute through a mechanism known as the autoregulation of cerebral perfusion (Fig. 1). This occurs despite wide fluctuations in the perfusion pressure in the range of 70-120 mm Hg. Any increase in pressure automatically results in vasoconstriction and any decrease in vasodilation. These responses lower the risk of cerebral hyper- and hypoperfusion respectively. After an acute ischemic stroke, the autoregulation of cerebral perfusion is lost in the tissues surrounding the ischemic core, the so-called penumbra. This peri-

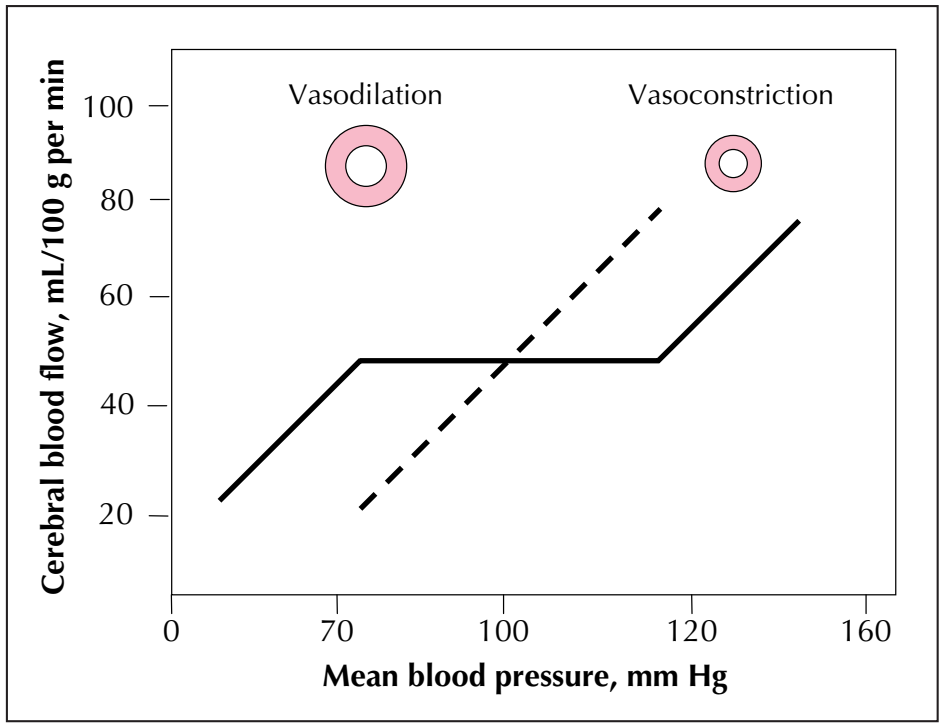

Fig. 1: Autoregulation of cerebral blood flow in a normal brain and in the ischemic penumbra (the tissues surrounding the ischemic core after a stroke). In the normal brain, cerebral blood flow is kept at $50 \mathrm{~mL} / 100 \mathrm{~g}$ per minute, despite continuous fluctuations of mean blood pressure between 70 and $120 \mathrm{~mm} \mathrm{Hg}$ (continuous line). Any increase in pressure leads to vasoconstriction and any decrease to vasodilation, which prevents the risk of cerebral hyper- and hypoperfusion, respectively. Above and below the limits of cerebral blood flow autoregulation, cerebral perfusion passively follows the perfusion pressure. In the ischemic penumbra, tissue perfusion follows perfusion pressure (dashed line): any fall in blood pressure may precipitate ischemia, while an increase in blood pressure may cause edema and hemorrhagic transformation. 
infarct zone is a moderately ischemic area affected by varying degrees of injury. The area may be salvaged if blood flow is rapidly restored within hours after the initial injury because, although electrical function has been lost, the ionic pumps have not yet failed. Flow in the range of $10-20 \mathrm{~mL} / 100 \mathrm{~g}$ per minute is the border between irreversible and reversible damage. Because of the loss of autoregulation in the penumbra, the extent of cerebral perfusion depends on the perfusion pressure, and a fall in blood pressure during this critical time may reduce cerebral perfusion, extend the ischemic area, induce irreversible damage and worsen the disabling consequences of the initial stroke. Therefore, during the first 24-48 hours a high blood pressure may be desirable to reduce the cerebral damage, until the autoregulation is restored and any further neurologic improvement unlikely. In contrast, in the later phase a smooth rate of blood pressure reduction is recommended, in order to reduce the risk of cerebral edema, hemorrhagic transformation, stroke recurrence and cardiovascular complications.

Unfortunately, the impact of blood pressure changes on cerebral perfusion in acute ischemic stroke is difficult to anticipate. Emboli may be dissolved and fragmented by intrinsic thrombolytic mechanisms, migrate to distal branches and finally disappear within hours or days. High perfusion pressure in this situation may result in luxury perfusion (abnormally increased flow of blood to an area, causing edema) of the previously ischemic area, cerebral edema and hemorrhagic transformation. The administration of a thrombolytic agent may be ineffective, leaving uncertainty about whether the artery has been successfully reopened or not. If reopened, there is risk of luxury perfusion, cerebral edema and hemorrhagic transformation at high perfusion pressure. On the other hand, if thrombolysis was ineffective in reopening the vessel, the low blood pressure required for a safe intravenous thrombolysis may induce cerebral hypoperfusion and extend the ischemic core.

Given these uncertainties, the American Stroke Association and the European Stroke Initiative recommend that only patients with blood pressure values repeatedly above $220 / 120 \mathrm{~mm} \mathrm{Hg}$ should be given either labetalol or sodium nitroprusside, intravenously, unless there are other indications for antihypertensive therapy (congestive heart failure, myocardial infarction, aortic dissection). ${ }^{4}$ The blood pressure target during the acute phase of an ischemic stroke should not be a normal blood pressure but, rather, 180/105 mm Hg in previously hypertensive patients and 160-180/90-100 mm Hg in previously normotensive patients.

In patients who were not receiving antihypertensive treatment before the ischemic stroke and who have a baseline systolic pressure of 180-220 mm Hg and a diastolic pressure below $120 \mathrm{~mm} \mathrm{Hg}$, antihypertensive therapy should be deferred for the first 48 hours after the stroke, unless thrombolytic therapy is indicated. In patients who were already receiving oral antihypertensive therapy before the stroke and who have a baseline blood pressure within the above-mentioned range, antihypertensive therapy should be given to avoid rebound hypertension, with the aim of maintaining a systolic pressure of 180-220 $\mathrm{mm} \mathrm{Hg}$ and a diastolic pressure below $120 \mathrm{~mm} \mathrm{Hg}$. If the systolic pressure is higher than $220 \mathrm{~mm} \mathrm{Hg}$ and the diastolic pressure higher than $120 \mathrm{~mm} \mathrm{Hg}$, intravenous antihypertensive drugs are recommended to keep the blood pressure at about $180 / 100-105 \mathrm{~mm} \mathrm{Hg}$. It is important to select rapidly reversible agents in case neurologic signs and symptoms worsen with the blood pressure reduction.

Patients, families, emergency physicians and paramedics alike should be instructed to abstain from administering antihypertensive agents if stroke symptoms are present, until the diagnosis of stroke has been established and the existence of severe hypertension requiring treatment confirmed. In the acute phase of stroke they must resist the temptation to "catch up" on failed prevention by starting or potentiating antihypertensive therapy too early, since by this action they may unwittingly interfere with cerebral perfusion of the injured brain.

\section{Andrea Semplicini \\ Lorenzo Calò \\ Department of Clinical \\ and Experimental Medicine \\ University of Padua Medical School \\ Padua, Italy}

\section{References}

1. Leonardi-Bee J, Bath PM, Phillips SJ, Sandercock PA; IST Collaborative Group. Blood pressure and clinical outcomes in the International Stroke Trial. Stroke 2002;33(5):1315-20.

2. Castillo J, Leira R, Garcia MM, Serena J, Blanco M, Davalos A. Blood pressure decrease during the acute phase of ischemic stroke is associated with brain injury and poor stroke outcome. Stroke 2004;35(2):520-6.

3. Semplicini A, Maresca A, Boscolo G, Sartori M, Rocchi R, Giantin V, et al. Hypertension in acute ischemic stroke: A compensatory mechanism or an additional damaging factor? Arch Intern Med 2003;163(2):211-6.

4. Klijn CJ, Hankey GJ; American Stroke Association and European Stroke Initiative. Management of acute ischaemic stroke: new guidelines from the American Stroke Association and European Stroke Initiative. Lancet Neurol 2003;2(11):698-701. 